Bayot, A., Hernández Viadel, J. V. y de Julian, Luis Felipe (2005): Análisis factorial exploratorio y propiedades psicométricas de la escala de competencia parental percibida. Versión para padres/madres (ECPP-p). RELIEVE: v. 11, n. 2, p. 113-126. http://www.uv.es/RELIEVE/v11n2/RELIEVEv11n2 2.htm

\title{
Re्IIEVद
}

Revista ELectrónica de Investigación

y EValuación Educativa

\section{ANÁLISIS FACTORIAL EXPLORATORIO Y PROPIEDADES PSICOMÉTRICAS DE LA ESCALA DE COMPETENCIA PARENTAL PERCIBIDA. VERSIÓN PARA PADRES/MADRES (ECPP-p) \\ (Exploratory factorial analysis and psychometrics properties on the Scale of Sensed Parental Competencies. Version for parents (ECPP-p).)}

por

Article record

About authors

$\underline{\text { HTML format }}$

\author{
Agustín Bayot (agustin.bayot@uclm.es) \\ José Vicente Hernández Viadel \\ Luis Felipe de Julián
}

$\underline{\text { Ficha del artículo }}$
$\underline{\text { Sobre los autores }}$
Formato HTML

\begin{abstract}
The lack of parental (or relative) competence is related to the development of a model of unsuitable behaviour in children (sons or daughters), suffering the consequences of this fact. However, practically there is no existence of evidences that can measure this. The Scale of Parental Competence Sensed (parents vision) (ECPP$\mathrm{P})$ it is composed by twenty-two items which are structured in five factors. The ECPP shows some psychometric properties highly acceptables.
\end{abstract}

\section{Keywords}

Parental competence, familiar interaction, parental satisfaction, problematic behaviours

\section{Resumen}

La escasa competencia parental está relacionada con el desarrollo de un patrón de conducta desadaptativo en los/as hijos/as, con las consecuencias que este hecho conlleva. Sin embargo, prácticamente no existen pruebas que midan este constructo. La Escala de Competencia Parental Percibida (versión para padres/madres)(ECPP-p) está constituida por 22 items, que se estructuran en cinco factores. La ECPP presenta unas propiedades psicométricas altamente aceptables

\section{Descriptores}

Competencia parental, interacción familiar, satisfacción parental, conductas problemáticas

\section{INTRODUCCIÓN}

La responsabilidad de educar a los/as hijos/as ha recaído durante muchos años en grupos familiares ligados a ellos por lazos de afiliación, de alianza y de cohabitación. La responsabilidad de educar a los pequeños co- rrespondía entonces exclusivamente a los/as padres/madres y a quienes les rodeaban.

Progresivamente, la escuela y otros agentes educativos han ido asumiendo la tarea y la responsabilidad de satisfacer las necesidades que plantea el desarrollo de los/as niños/as y de preparar su futuro en el seno de la socie- 
dad. Por otra parte, cuando en determinadas familias con hijos/as han existido dificultades para el buen desarrollo físico, psicológico y social de los menores, los poderes públicos han confiado esta tarea a otras familias de acogida o a centros encargados de ejercer las responsabilidades educativas y socializadoras.

Debido a lo anteriormente expuesto, vemos necesario que, desde el ámbito de los profesionales que trabajamos en torno al bienestar y desarrollo de la familia y de sus miembros, se produzcan investigaciones y desarrollos teóricos que estudien a la familia desde todas sus dimensiones y, sobretodo, que profundicen en las aptitudes, actitudes, cualidades y comportamientos que los padres y las madres realizan habitualmente y como este proceso ejerce su influencia en los comportamientos futuros y en la conformación de la personalidad de sus hijos/as. Si todo esto se llevara a cabo, encontraríamos el camino para mejorar las actitudes de los/as padres/madres y, sobretodo desde otras intervenciones sociales, educativas, etc., se podría formar, ayudar y apoyar a los/as padres/madres y a las familias en sus tareas educativas con los/as niños/as.

Si acudimos a la literatura especializada, encontramos referencias relativas a que la génesis de determinados comportamientos psicopatológicos de los individuos, así como conductas socialmente inadaptadas y desviadas de la norma social establecida, se encuentran en el núcleo familiar (Musitu y Cava, 2001). Las relaciones que mantienen los/as padres/madres con los/as hijos/as son fundamentales en la educación que éstos reciben (Aguilar Ramos, 2002). Concretamente, en una serie de investigaciones se ha puesto de manifiesto que las relaciones familiares positivas y una alta comunicación entre los/as padres/madres y los/as hijos/as se relaciona con un bajo consumo de drogas y la aparición de conductas antisociales en la adolescencia (Villar, Luengo, Gómez y Romero, 2003), la asociación entre patrones parentales y las prácticas de riesgo (Martínez, Fuertes, Ramos y Hernández, 2003), etc.
Hay que señalar que una de las preocupaciones más relevantes de los investigadores radica en determinar cómo las actitudes y comportamientos que los/as padres/madres mantienen en su relación diaria con sus hijos/as van a influir directamente en su personalidad y su conducta futura. Ya en 1976 Eysenck planteó cómo las variables personales del individuo ejercen una gran influencia sobre la modulación del estrés familiar y el tipo de conducta social de los individuos.

Detectar tempranamente factores como el tipo y modalidad de la interacción familiar y la competencia parental percibida ha mostrado su relevancia a la hora de elaborar modelos explicativos de la función parental.

Sin embargo, nos encontramos con que la casi totalidad de las investigaciones se centran en la relación existente entre las prácticas de socialización familiar disfuncionales, tales como disciplina punitiva, y el desarrollo de problemas de conducta en niños (Cerezo, Keesler, Dunn y Wahler 1991; Wahler, Williams y Cerezo, 1990).

En nuestro trabajo, queremos hacer hincapié, a través de la construcción de la Escala de Competencia Parental Percibida (versión para padres/madres)(ECPP-p), en la detección de algunos de los factores que pueden influir positivamente en una satisfacción parental percibida por parte de los/as padres/madres de manera que permitan en el futuro plantear abordajes preventivos de malos tratos infantiles, por un lado, y conseguir que los/as padres/madres incrementen su satisfacción con la función diaria de ser y ejercer como tales, por otro.

Para ello, hemos realizado una revisión de los instrumentos desarrollados en este campo. Siguiendo a Grisso (1986), se pueden clasificar en dos grupos: 1) los elaborados para evaluar aptitudes, conductas y habilidades parentales y, 2) los que se centran en la identificación de progenitores en riesgo de maltratar a sus hijos/as. Obviamente, nuestro trabajo se centra en el primer grupo. 
Fariña, Seijo, Novo y Jólluskin (2002) encuentran 17 escalas que cumplen las mismas características que las de Grisso.

En otra revisión realizada por Holden y Edwards (1989), se señalan graves problemas metodológicos con respecto a las investigaciones que han elaborado cuestionarios sobre estilos educativos parentales. Los problemas van desde que se evalúan intenciones u opiniones de los/as padres/madres, los items están formulados de forma muy genérica, etc. Este hecho provoca que los/as padres/madres contesten más por lo que se espera de ellos, que por sus prácticas concretas de crianza como padres y madres.

Dentro de este grupo de escalas, algunas resultan más afines a nuestra investigación, ya que evalúan las creencias y opiniones que los/as padres/madres presentan acerca de su capacidad parental. Entre ellas, cabe mencionar la Parental Attitude Survey-PAS (Heredorf, 1963), formada por 5 escalas: a) confianza en el rol parental, b) causación de la conducta del niño, c) aceptación, d) comprensión mutua y e) confianza mutua.

Este tipo de herramientas suelen ser utilizadas habitualmente, en el ámbito anglosajón, en el ámbito de la Psicología forense y cuando han sido adaptadas a nuestro territorio denotan multitud de carencias y limitaciones. Respecto de los aspectos metodológicos, se detectan determinadas carencias debido a su excesiva longitud, no se aporta información sobre sus propiedades psicométricas, etc.

Esta situación ha obligado a una serie de investigadores a adaptar en nuestro país los cuestionarios validados en otras poblaciones (Castro, Toro, Van Der Ende y Arrindel, 1993; Gracia y Musitu, 1993, Roa y del Barrio, 2001, etc.).

Concretamente, Roa y del Barrio (2001) han adaptado el Cuestionario de Crianza Parental (PCRI -Gerard, 1998-), donde se miden las actitudes de los/as padres/madres hacia la crianza de los/as hijos/as. Esta escala está dividida en 8 subescalas: apoyo, satisfacción con la crianza, compromiso, comunicación, disciplina, autonomía, distribución del rol y deseabilidad social.

Concretamente en nuestro país, se han elaborado una serie de escalas que miden los estilos educativos de los/as padres/as. Dentro de este grupo, es importante reseñar el Perfil de Estilos Educativos (PEE, Lago y García, 1998). Este instrumento posibilita la identificación del estilo educativo, relacionado con las ideas, creencias, actitudes y valores sobre la educación de los/as hijos/as y con los/as alumnos/as, ya que existen dos versiones: una dirigida a los/as padres/madres (PPE-pd) y otra a los/as profesores/as (PPE-pd).

Además, también cabe mencionar la Escala de Evaluación de Estilos Educativos (4E) (Palacios, 1994) y el Cuestionario Situacional de Metas y Prácticas Educativas (Ceballos y Rodrigo, 1992).

Fuentes, Motrico y Bersabé (1999) han elaborado la Escala de Normas y Exigencias (ENE) y la Escala de Afecto (EA). Estas escalas están midiendo los estilos educativos parentales basados en las normas (Bersabé, Fuentes y Motrico, 2001).

Sin embargo, son prácticamente inexistentes las pruebas que miden la competencia parental en situaciones concretas.

Las conclusiones más relevantes que se pueden extraer a partir de las revisiones realizadas son las siguientes: a) inexistencia de cuestionarios que permitan la determinación de la competencia parental percibida en situaciones concretas; b) no existe ningún instrumento elaborado con población española, y c) los instrumentos analizados cuentan con limitaciones, fundamentalmente de índole psicométrico.

Por tanto, los motivos por los que hemos elaborado la ECPP-p son: validar una escala de competencia parental, versión para padres y madres, conseguir un mayor conocimiento de los estilos parentales en situaciones concretas, plantear estrategias de intervención de carácter preventivo así como diseñar líneas futuras de 
investigación con la finalidad de conocer las funciones parentales adecuadas que determinan un estilo educativo óptimo.

\section{METODOLOGÍA}

\section{II.1. Proceso de elaboración de la escala}

La Escala de Competencia Parental Percibi$\mathrm{da}^{[1]}$, en su versión para padres y madres (ECPP-p), consta de 22 items. A partir de la revisión de la literatura, se construyeron 122 items relacionados con la participación activa de los/as padres/madres en las actividades escolares de sus hijos/as (Musitu y Cava, 2001), el tiempo que los/as padres/madres dedican a sus hijos/as de una forma constructiva (Molpeceres, Musitu y Lila, 1994), la dedicación personal de los/as padres/madres, la función socializadora de la familia, la comunicación familiar (Baer y Bray, 1999), asumir la responsabilidad que entraña el hecho de ser padres (Inglés, 1995; Moreno, Jiménez, Oliva, Palacios y Saldaña, 1995), etc.

Posteriormente, se llevó a cabo el estudio piloto con la finalidad de depurar la longitud de la escala. En dicha depuración, se han tenido en cuenta los siguientes criterios: a) redundancia de los items respecto de la escala de respuesta; b) índice de asimetría y de curtosis de los items; c) valoración de un grupo de expertos sobre si los items están midiendo el constructo "competencia parental"; d) items con contenido igual o similar, y e) diferencias en función del sexo.

A partir de los criterios descritos con anterioridad, la versión original de la ECPP-p quedó configurada por 56 items.

El pase definitivo de la escala se llevó a cabo con una muestra de 1074 participantes $(\mathrm{N}=$ 1074). A partir de los análisis psicométricos, la ECPP está constituida por 22 items.

Los participantes deben expresar el grado en el que están de acuerdo, con cada uno de los 22 items, siguiendo una escala tipo Likert, de cuatro alternativas de respuesta, en la que (1) es "si no le ocurre NUNCA o muy rara vez";
(2) "si le ocurre A VECES o de vez en cuando"; (3) "si le ocurre CASI SIEMPRE", y 4) "si le ocurre SIEMPRE".

\section{II.2. Descripción de la muestra}

\section{Para el estudio piloto}

La versión original de la Escala de Competencia Parental Percibida fue cumplimentada por una muestra de 171 participantes, de los cuales el $33.3 \%$ pertenecen a mujeres, el $33.3 \%$ a hombres y el 33.3 restante a parejas. La media de edad de todos los participantes es de 39.84 años $(\mathrm{X}=39.84$; $\mathrm{Sx}=5.27)$. El promedio de hijos/as es de $2.14(\mathrm{X}=2.14 ; \mathrm{Sx}=$ 1.00) y la edad en la que tuvieron el primer hijo/a fue de 27,90 años ( $X=27.90 ; \mathrm{Sx}=4.28)$.

\section{Para el pase definitivo de la escala}

La versión constituida por 56 items de la Escala de Competencia Parental Percibida fue cumplimentada por 1074 participantes $(\mathrm{N}=$ 1074) de diferentes provincias españolas. Entre las provincias en las que existe una mayor representatividad, se encuentran Valencia $(9,70 \%)$, Cuenca $(10,00 \%), \quad$ Guadalajara $(12,80 \%)$, Madrid (31,20\%), etc.

Del total de la muestra, el $35,60 \%$ corresponde a mujeres, el $32,50 \%$ a hombres y el $31,90 \%$ a parejas. Con relación al estado civil, el $92,20 \%$ está casado y el 7,87\% restante está separado, soltero, divorciado, viudo o conviviendo como pareja de hecho.

La media de edad de todos los participantes es de 39,32 años $(X=39,32 ; \mathrm{Sx}=5,50)$; en promedio, las mujeres tuvieron el primer hijo/a a los 26,67 años $(X=26,67 ; S x=4,51)$ y los/as padres/madres a los 29,04 años $(\mathrm{X}=$ 29,04; $\mathrm{Sx}=4,80)$. El promedio de hijos/as es de 2,00 años $(X=2,00 ; S x=0,78)$.

Respecto de los estudios cursados, el $38,50 \%$ corresponde a EGB, el $38,10 \%$ a estudios medios y el $20,00 \%$ a estudios superiores.

El 50,50\% trabaja en el sector servicios y el $21,30 \%$ realiza las funciones de ama de casa.

\section{II.3. Procedimiento}


Estudio piloto. La muestra estuvo formada por participantes de Castilla-La Mancha. Para ser seleccionados, tenían que reunir dos criterios: 1) tener hijos/as con edades comprendidas entre 3 y 18 años, y 2) ser usuarios de servicios sociales, y/o padres/madres de alumnos/as de centros educativos.

Pase definitivo de la prueba. En este caso, se siguieron los mismos criterios de selección de la muestra. Sin embargo, en esta ocasión, los participantes son representativos de todas las comunidades autónomas españolas.

El primer contacto se realizó por medio de una carta de presentación del equipo de investigación, en la que se describía a grandes rasgos el proyecto de investigación y se invitaba a los centros a que participaran en el estudio. Una semana después del envío de la carta, un componente del equipo de investigación se puso en contacto telefónicamente con los diferentes centros seleccionados. En los casos en los que mostraron su disponibilidad, se acordó el número de encuestas, así como la persona encargada del pase de las encuestas, a la que nos dirigimos posteriormente para evaluar el resultado del proceso.

A cada centro se le envió el número de cuestionarios pactados (en sus tres versiones: padres, madres y pareja), así como el material necesario para que nos reenviasen los cuestionarios cumplimentados de forma gratuita. También se adjuntó una nota de agradecimiento por la colaboración y un documento donde se justificase que los cuestionarios saliesen del centro, con la firma del responsable del mismo.

Por medio del programa de análisis de datos SPSS (versión 11,00 para Windows), se realizó un análisis factorial exploratorio, siguiendo el principio de Kaiser, mediante la técnica de componentes principales y rotación varimax. La consistencia interna de la escala se calculó a partir del coeficiente alfa de Cronbach. Además, también se hallaron otras medidas estadísticas, así como una serie de pruebas de diferencias en función de los estudios realizados, tipo de entrevista y profesión.

\section{RESULTADOS}

\section{III.1. Coeficiente de homogeneidad}

Todos los items que conforman la Escala de Competencia Parental Percibida, mantienen una correlación corregida elevada con la puntuación total de la escala (tabla 1), con un rango que oscila entre 0,22 (item 22: "soy muy consciente del cambio que ha experimentado mi familia con la llegada de los/as hijos/as") y 0,60 (item 4: "consulto con el/la maestro/a la evolución de mis hijos/as en el colegio").

\section{III.2. Consistencia interna}

Por lo que respecta a la fiabilidad total de la escala, el coeficiente Alpha de Cronbach es de $0,86(\alpha=0,86)$ (tabla 1$)$, lo que indica que posee una adecuada consistencia interna. Con relación a cada uno de los factores, los resultados fueron los siguientes: implicación escolar $(\alpha=0,76)$, dedicación personal $(\alpha=0,68)$, ocio compartido $(\alpha=0,56)$, asesoramiento/orientación $(\alpha=0,62)$ y asunción del rol de ser padre/madre $(\alpha=0,52)$.

\section{III.3. Análisis factorial}

A partir del análisis de componentes principales llevado a cabo con los 22 items de la Escala de Competencia Parental Percibida, se han obtenido 5 componentes en la solución final, por medio de la rotación varimax. Los cinco componentes explican el 48,80\% de la varianza total, con saturaciones que oscilan entre 0,48 y 0,69 (tabla 2).

El primer componente, con un valor propio de 2,67 , explica el $12,14 \%$ de la varianza explicada. Incluye los items, de mayor a menor saturación, 11, 21, 4, 13 y 15 . Atendiendo a su contenido, este factor está midiendo la "IMPLICACIÓN ESCOLAR” de los/as padres/madres. Recibe este nombre, ya que mide el grado de preocupación y de participación que se da en el momento de responder a la escala a los aspectos escolares de los/as hijos/as.

El segundo componente, con un valor propio de 2,46 , explica el $11,19 \%$ de la varianza explicada. Está formado por los items 10, 12, 9, 
Bayot, A., Hernández Viadel, J. V. y de Julian, Luis Felipe (2005): Análisis factorial exploratorio y propiedades psicométricas de la escala de competencia parental percibida. Versión para padres/madres (ECPP-p). RELIEVE: v. 11, n. 2, p. 113-126. http://www.uv.es/RELIEVE/v11n2/RELIEVEv11n2 2.htm

5 y 20. Está midiendo la "DEDICACIÓN PERSONAL". Esta subescala evalúa en qué medida los/as padres/madres dedican sus tiempos y espacios para conversar, explicar dudas, transmitir valores; en definitiva, para "estar con ellos de una manera constructiva".

Tabla 1. Homogeneidad corregida de los items y Alpha del cuestionario excluido el item.

\begin{tabular}{|c|c|c|c|c|}
\hline ITEMS & $\mathrm{X}$ & $\mathrm{Sx}$ & $\mathrm{CH}-1$ & $\alpha-1$ \\
\hline (1) Felicito a mis hijos/as cada vez que hacen algo bien. & 3,63 & 0,59 & 0,35 & 0,86 \\
\hline (2) Respaldo en casa las reglas0, normas y expectativas de conducta de la escuela. & 3,52 & 0,65 & 0,36 & 0,86 \\
\hline (3) En casa se fomenta que cada uno exprese sus opiniones. & 3,43 & 0,68 & 0,37 & 0,85 \\
\hline (4) Consulto con el/la maestro/a la evolución de mis hijos/as en el colegio. & 3,18 & 0,96 & 0,60 & 0,85 \\
\hline (5) Conozco los deberes y tareas que les ponen en el colegio a mis hijos/as. & 3,35 & 0,81 & 0,56 & 0,85 \\
\hline $\begin{array}{l}\text { (6) Acudo a lugares donde hay más niños/as para potenciar que mis hijos/as se rela- } \\
\text { cionen. }\end{array}$ & 2,99 & 0,85 & 0,44 & 0,85 \\
\hline $\begin{array}{l}\text { (7) Veo con mis hijos/as ciertos programas de TV y los comento con ellos/as des- } \\
\text { pués. }\end{array}$ & 2,56 & 0,79 & 0,30 & 0,86 \\
\hline (8) Me preocupo por incluir a mis hijos/as en actividades extraescolares. & 3,18 & 0,89 & 0,42 & 0,85 \\
\hline (9) Colaboro en las tareas del hogar. & 3,35 & 0,89 & 0,46 & 0,85 \\
\hline $\begin{array}{l}\text { (10) Ayudo a mis hijos/as a establecer una rutina diaria en cuanto a hábitos de higie- } \\
\text { ne. }\end{array}$ & 3,55 & 0,69 & 0,55 & 0,85 \\
\hline $\begin{array}{l}\text { (11) Mantengo un contacto frecuente con la escuela para estar al tanto de cualquier } \\
\text { información referente a los programas y recursos para las familias. }\end{array}$ & 2,71 & 0,96 & 0,53 & 0,85 \\
\hline $\begin{array}{l}\text { (12) Mantengo organizado una especie de archivo de mis hijos/as donde se incluyan: } \\
\text { datos médicos, escolares, fotos, documentación legal, etc. }\end{array}$ & 3,17 & 0,99 & 0,41 & 0,85 \\
\hline $\begin{array}{l}\text { (13) Tengo una mentalidad positiva y abierta al conversar con el personal de la es- } \\
\text { cuela. }\end{array}$ & 3,30 & 0,78 & 0,57 & 0,85 \\
\hline $\begin{array}{l}\text { (14) Respondo a las encuestas escolares que busquen averiguar los intereses, talento } \\
\text { y habilidades de mis hijos/as. }\end{array}$ & 3,27 & 0,86 & 0,41 & 0,85 \\
\hline (15) Establezco conjuntamente con el maestro/a, las metas y expectativas del niño/a. & 2,52 & 1,00 & 0,53 & 0,85 \\
\hline $\begin{array}{l}\text { (16) Existe una hora fija en la que mis hijos/as tengan que estar acostados o levanta- } \\
\text { dos. }\end{array}$ & 3,01 & 0,89 & 0,29 & 0,86 \\
\hline (17) Dedico un tiempo al día para hablar con mi hijo/a. & 3,03 & 0,81 & 0,50 & 0,85 \\
\hline (18) Ayudo a mis hijos/as a establecer una rutina diaria en lo referido al estudio. & 3,19 & 0,84 & 0,56 & 0,85 \\
\hline (19) Hago pequeñas excursiones con la familia al cine, zoo, museos, parques, etc. & 2,93 & 0,89 & 0,40 & 0,85 \\
\hline (20) Dispongo de suficiente tiempo para atender a mis hijos/as. & 2,91 & 0,90 & 0,42 & 0,85 \\
\hline $\begin{array}{l}\text { (21) Asisto regularmente a las reuniones que se establecen en la Asociación de Ma- } \\
\text { dres y Padres de Alumnos (AMPA). }\end{array}$ & 2,37 & 1,12 & 0,33 & 0,86 \\
\hline $\begin{array}{l}\text { (22) Soy muy consciente del cambio que ha experimentado mi familia con la llegada } \\
\text { de los/as hijos/as. }\end{array}$ & 3,56 & 0,66 & 0,22 & 0,86 \\
\hline
\end{tabular}

$X=$ Media; $S x=$ Desviación típica; $C H-1=$ Coeficiente de homogeneidad corregido de los items; $a-1=$ Alpha del cuestionario excluido el item.

El valor propio del tercer componente es de 2,14 , con un $9,73 \%$ de varianza explicada. Está compuesto por los items 7, 6, 19 y 8. Está midiendo el "OCIO COMPARTIDO". Es decir, plantea cómo se perciben los/as padres/madres en cuanto a si planifican el tiempo libre para realizar actividades en las que participan todos los miembros o, por el contrario, el tiempo libre es utilizado de manera individual.
El cuarto componente tiene un valor propio de 1,75 , con un $7,95 \%$ de varianza explicada. Los items son el 16, 14, 18 y 17. Está midiendo el "ASESORAMIENTO Y LA ORIENTACIÓN" que proporcionan los/as padres/madres a sus hijos/as. Destaca la capacidad de diálogo y escucha de los/as padres/madres a la hora de atender las demandas y necesidades de sus hijos/as.

Finalmente, el quinto componente presenta un valor propio de 1,72 y está explicando el 
Bayot, A., Hernández Viadel, J. V. y de Julian, Luis Felipe (2005): Análisis factorial exploratorio y propiedades psicométricas de la escala de competencia parental percibida. Versión para padres/madres (ECPP-p). RELIEVE: v. 11, n. 2, p. 113-126. http://www.uv.es/RELIEVE/v11n2/RELIEVEv11n2 2.htm

$7,80 \%$ de la varianza explicada. Está compuesto por los items 2, 22, 3 y 1 . Este factor está midiendo la "ASUNCIÓN DEL ROL DE SER PADRE/MADRE”. Es decir, en qué me- dida los progenitores se han adaptado a las circunstancias que conllevan el nacimiento de los/as hijos/as.

Tabla 2. Resultados del análisis de componentes principales realizados

\section{ITEMS}

(11) Mantengo un contacto frecuente con la escuela para estar al tanto de cualquier información referente a los programas y recursos para las familias.

(21) Asisto regularmente a las reuniones que se establecen en la Asociación de Madres y Padres de Alumnos (AMPA).

(4) Consulto con el/la maestro/a la evolución de mis hijos/as en el colegio.

(13) Tengo una mentalidad positiva y abierta al conversar con el personal de la escuela.

(15) Establezco conjuntamente con el maestro/a, las metas y expectativas del niño/a.

(10) Ayudo a mis hijos/as a establecer una rutina diaria en cuanto a hábitos de higiene.

(12) Mantengo organizado una especie de archivo de mis hijo/as donde se incluyan: datos médicos, escolares, fotos, documentación legal, etc.

(9) Colaboro en las tareas del hogar.

(5) Conozco los deberes y tareas que les ponen en el colegio a mis hijos/as.

(20) Dispongo de suficiente tiempo para atender a mis hijos/as.

(7) Veo con mis hijos/as ciertos programas de TV y los comento con ellos/as después.

(6) Acudo a lugares donde hay más niños/as para potenciar que mis hijos/as se relacionen.

(19) Hago pequeñas excursiones con la familia al cine, zoo, museos, parques, etc.

(8) Me preocupo por incluir a mis hijos/as en actividades extraescolares.

(16) Existe una hora fija en la que mis hijos/as tengan que estar acostados o levantados.

(14) Respondo a las encuestas escolares que busquen averiguar los intereses, talento y habilidades de mis hijos/as.

(18) Ayudo a mis hijos/as a establecer una rutina diaria en lo referido al estudio.

(17) Dedico un tiempo al día para hablar con mi hijo/a.

(2) Respaldo en casa las reglas, normas y expectativas de conducta de la escuela.

(22) Soy muy consciente del cambio que ha experimentado mi familia con la llegada de los/as hijos/as.

(3) En casa se fomenta que cada uno exprese sus opiniones.

(1) Felicito a mis hijos/as cada vez que hacen algo bien.

Autovalores*

Porcentaje de varianza

Coeficiente a

$F_{1}=$ Factor de "Implicación escolar"; $F_{2}=$ Factor de "Dedicación personal"; $F_{3}=$ Factor de "Ocio compartido", $F_{4}=$ Factor de "Asesoramiento/orientación" y $F_{5}=$ Factor de "Asunción del rol de ser padre/madre".

* En este caso, se toman los valores de las sumas de las saturaciones al cuadrado de la rotación.. 
Bayot, A., Hernández Viadel, J. V. y de Julian, Luis Felipe (2005): Análisis factorial exploratorio y propiedades psicométricas de la escala de competencia parental percibida. Versión para padres/madres (ECPP-p). RELIEVE: v. 11, n. 2, p. 113-126. http://www.uv.es/RELIEVE/v11n2/RELIEVEv11n2 2.htm

\section{III.4. Validez discriminante}

En este apartado nos centraremos en analizar las diferencias que puedan existir en función del estado civil, el tipo de encuesta cumplimentada, la profesión y los estudios cursados, respecto de cada uno de los cinco factores que está formada la ECPP-p.

\section{III.4.1. Diferencias en función del estado civil}

Con relación al estado civil, únicamente se observan diferencias estadísticamente significativas en el factor "asunción del rol de ser padre/madre" $\{\mathrm{F}(2,1051)=3,27 ; \mathrm{p}<0,05)$ (tabla 3). No obstante, aunque en la F Ratio aparecen diferencias significativas, en la prueba de Tukey aplicada no se obtienen diferencias significativas entre las diferentes comparaciones realizadas.

Tabla 3. Estado civil

\begin{tabular}{|c|c|c|c|c|c|c|c|c|c|}
\hline & \multicolumn{2}{|l|}{ Casado } & \multicolumn{2}{|c|}{ Separado } & \multicolumn{2}{|c|}{ Soltero } & \multirow{2}{*}{$\mathrm{F}$} & \multirow{2}{*}{$\mathrm{p}$} & \multirow{2}{*}{$\eta^{2}$} \\
\hline Variables & $\mathrm{X}$ & Sx & $\mathrm{X}$ & $\mathrm{Sx}$ & $\mathrm{X}$ & Sx & & & \\
\hline $\mathrm{F}_{1}$ & 14,18 & 3,50 & 13,62 & 2,07 & 13,79 & 3,11 & 0,58 & 0,55 & 0,001 \\
\hline $\mathrm{F}_{2}$ & 16,35 & 2,89 & 16,03 & 2,51 & 16,41 & 2,66 & 0,18 & 0,83 & 0,0005 \\
\hline $\mathrm{F}_{3}$ & 11,69 & 2,25 & 11,27 & 2,25 & 12,00 & 2,28 & 0,86 & 0,42 & 0,002 \\
\hline $\mathrm{F}_{4}$ & 12,51 & 2,34 & 12,75 & 1,80 & 12,45 & 2,41 & 0,17 & 0,84 & 0,0005 \\
\hline $\mathrm{F}_{5}$ & 14,19 & 1,64 & 13,75 & 1,37 & 13,60 & 2,23 & 3,27 & 0,04 & 0,006 \\
\hline
\end{tabular}

$X=$ Media $; S_{X}=$ Desviación típica $; \mathrm{F}=$ Prueba de contraste de hipótesis $\mathrm{F}$ Ratio; $\mathrm{p}=$ probabilidad; $\eta^{2}=$ tamaño del efecto; $F_{1}=$ Factor de "Implicación escolar"; $F_{2}=$ Factor de "Dedicación personal"; $F_{3}=$ Factor de "Ocio compartido"; $F_{4}=$ Factor de “Asesoramiento/orientación” y $F_{5}=$ Factor de "Asunción del rol de ser padre/madre”.

\section{III.4.2. Diferencias entre el padre, la ma- dre y la pareja}

Con relación a si la ECPP-p la cumplimentan los/as padres/madres o la pareja, existen diferencias significativas en "implicación escolar" $\{\mathrm{F}(2,1058)=115,47 ; \mathrm{p}<0,0005\}$, "dedicación personal" $\{\mathrm{F}(2,1060)=180,90 ; \mathrm{p}<$ $0,0005\}$, "ocio compartido" $\{\mathrm{F}(2,1062)=$ $16,31 ; \quad \mathrm{p}<0,0005\}$, "asesoramiento/orientación” $\quad\{\mathrm{F}(2,1054)=31,03 ; \quad \mathrm{p}<$
$0,0005\}$ y en "asunción del rol de ser padre/madre" $\quad\{\mathrm{F}(2,1065)=10,85 ; \quad \mathrm{p}<$ $0,0005\}$ (tabla 4). Las pruebas a posteriori aplicadas indican que las mujeres obtienen puntuaciones significativamente superiores en los cinco factores, con relación a los hombres y con relación a las parejas. Sin embargo, en el factor 3, las parejas obtienen puntuaciones superiores a los hombres (tabla 4).

Tabla 4. Tipo de encuesta cumplimentada

\begin{tabular}{|c|c|c|c|c|c|c|c|c|c|}
\cline { 2 - 8 } \multicolumn{1}{c|}{} & \multicolumn{2}{c|}{ Madre } & \multicolumn{2}{c|}{ Padre } & \multicolumn{2}{c|}{ Pareja } & \multirow{2}{*}{$\mathrm{F}$} & \multirow{2}{*}{$\eta^{2}$} \\
\hline Variables & $\mathrm{X}$ & $\mathrm{Sx}$ & $\mathrm{X}$ & $\mathrm{Sx}$ & $\mathrm{X}$ & $\mathrm{Sx}$ & & & \\
\hline $\mathrm{F}_{1}{ }^{\mathrm{AC}}$ & 15,24 & 2,81 & 11,99 & 3,79 & 15,00 & 2,77 & 115,47 & 0,0005 & 0,18 \\
\hline $\mathrm{F}_{2}{ }^{\mathrm{ABC}}$ & 17,80 & 1,87 & 14,35 & 3,21 & 16,74 & 2,21 & 180,90 & 0,0005 & 0,25 \\
\hline $\mathrm{F}_{3}{ }^{\mathrm{AC}}$ & 11,96 & 2,24 & 11,10 & 2,36 & 11,91 & 2,08 & 16,31 & 0,0005 & 0,03 \\
\hline $\mathrm{F}_{4}{ }^{\mathrm{AC}}$ & 13,05 & 2,09 & 11,74 & 2,58 & 12,68 & 2,13 & 31,03 & 0,0005 & 0,06 \\
\hline $\mathrm{F}_{5}{ }^{\mathrm{AC}}$ & 14,42 & 1,48 & 13,85 & 1,89 & 14,18 & 1,57 & 10,85 & 0,0005 & 0,02 \\
\hline
\end{tabular}

$X=$ Media; $S x=$ Desviación típica; $\mathrm{F}=$ Prueba de contraste de hipótesis $\mathrm{F}$ Ratio; $\mathrm{p}=$ probabilidad; $\eta^{2}=$ tamaño del efecto; $F_{1}=$ Factor de "Implicación escolar"; $F_{2}=$ Factor de "Dedicación personal"; $F_{3}=$ Factor de "Ocio compartido"; $F_{4}=$ Factor de "Asesoramiento/orientación” y $F_{5}=$ Factor de "Asunción del rol de ser padre/madre".

Prueba de Tukey: ${ }^{A}$ Mujer-hombre; ${ }^{B}$ Mujer-pareja; ${ }^{C}$ Hombre-pareja.

\section{III.4.3. Diferencias en función de la pro- fesión}

Respecto de la profesión de los participantes, aquélla se ha agrupado en seis categorías diferentes: obrero, sector servicios, profesión liberal, otro tipo de trabajos no incluidos en el resto, ama de casa y sin empleo. En los análisis estadísticos realizados, se han obtenido diferencias significativas en "implica- 
Bayot, A., Hernández Viadel, J. V. y de Julian, Luis Felipe (2005): Análisis factorial exploratorio y propiedades psicométricas de la escala de competencia parental percibida. Versión para padres/madres (ECPP-p). RELIEVE: v. 11, n. 2, p. 113-126. http://www.uv.es/RELIEVE/v11n2/RELIEVEv11n2 2.htm

ción escolar" $\{\mathrm{F}(5,680)=23,77 ; \mathrm{p}<0,0005\}$, "dedicación personal" $\{\mathrm{F}(5,681)=29,54 ; \mathrm{p}<$ $0,0005\}$, "ocio compartido" $\{\mathrm{F}(5,682)=6,01$; $\mathrm{p}<0,0005\}$, "asesoramiento/orientación" $\{\mathrm{F}(5,677)=5,26 ; \mathrm{p}<0,0005\}$ y en "asunción del rol de ser padre/madre" $\{\mathrm{F}(5,684)=4,99$; $\mathrm{p}<0,0005\}$ (tabla 5). Los resultados obtenidos en la prueba de Tukey indican que, prácticamente en todos los casos, las amas de casa obtienen puntuaciones superiores al resto de profesiones analizadas (tabla 5).

\section{Tabla 5. Profesión}

\begin{tabular}{|c|c|c|c|c|c|c|c|c|c|c|c|c|c|c|c|}
\hline & \multicolumn{2}{|c|}{ Obrero } & \multicolumn{2}{|c|}{ Servicios } & \multicolumn{2}{|c|}{ Prof. Lib. } & \multicolumn{2}{|c|}{ Otros } & \multicolumn{2}{|c|}{ Am. casa } & \multicolumn{2}{|c|}{ Sin empl. } & \multirow{2}{*}{$\mathrm{F}$} & & \multirow{2}{*}{$\eta^{2}$} \\
\hline Variables & $\mathrm{X}$ & $\mathrm{Sx}$ & $\mathrm{X}$ & $\mathrm{Sx}$ & $\mathrm{X}$ & $\mathrm{Sx}$ & $X$ & Sx & $\bar{X}$ & Sx & $X$ & Sx & & & \\
\hline $\begin{array}{c}\mathrm{F}_{1} \mathrm{ABCD} \\
\mathrm{EFGH}^{\mathrm{A}}\end{array}$ & 10,93 & 3,91 & 13,67 & 3,48 & 13,64 & 3,84 & 12,69 & 3,64 & 15,77 & 2,74 & 12,35 & 3,80 & 23,77 & 0,0005 & 0,15 \\
\hline $\begin{array}{c}\mathrm{F}_{2}{ }^{\mathrm{ADEFG}} \\
\mathrm{H}\end{array}$ & 14,64 & 2,88 & 15,74 & 3,09 & 14,28 & 3,63 & 15,44 & 3,46 & 18,50 & 1,64 & 16,30 & 2,25 & 29,54 & 0,0005 & 0,18 \\
\hline $\begin{array}{c}\mathrm{F}_{3}{ }^{\mathrm{DEHIJ}} \\
\mathrm{KL}\end{array}$ & 10,95 & 2,53 & 11,54 & 2,18 & 11,39 & 2,65 & 11,66 & 2,58 & 12,23 & 2,19 & 9,85 & 2,66 & 6,01 & 0,0005 & 0,04 \\
\hline$\overline{F_{4}{ }^{A D F}}$ & 11,64 & 2,83 & 12,46 & 2,29 & 11,39 & 2,51 & 12,55 & 2,41 & 12,99 & 2,26 & 11,55 & 2,48 & 5,26 & 0,0005 & $\overline{0,04}$ \\
\hline $\mathrm{F}_{5}{ }^{\mathrm{DEF}}$ & 13,90 & 1,84 & 14,06 & 1,67 & 13,28 & 2,12 & 14,34 & 170 & 14,63 & 1,35 & 13,60 & 2,60 & 4,99 & 0,0005 & 0,03 \\
\hline
\end{tabular}

$X=$ Media; $S x=$ Desviación típica; $\mathrm{F}=$ Prueba de contraste de hipótesis $\mathrm{F}$ Ratio; $\mathrm{p}=$ probabilidad; $\eta^{2}=$ tamaño del efecto; $F_{1}=$ Factor de "Implicación escolar"; $F_{2}=$ Factor de "Dedicación personal"; $F_{3}=$ Factor de "Ocio compartido"; $F_{4}=$ Factor de "Asesoramiento/orientación" y $F_{5}=$ Factor de "Asunción del rol de ser padre/madre". Prueba de Tukey: ${ }^{A}$ Obrero-servicios; ${ }^{B}$ Obrero-profesión liberal; ${ }^{C}$ Obrero-otros; ${ }^{D}$ Obrero-ama de casa; ${ }^{E}$ Servicios-ama de casa; ${ }^{F}$ Profesión liberal-ama de casa; ${ }^{G}$ Otros-ama de casa; ${ }^{H}$ Ama de casa-sin empleo; ${ }^{I}$ Serviciossin empleo; ${ }^{J}$ Otros-sin empleo; ${ }^{K}$ Sin empleo-servicios; ${ }^{L}$ Sin empleo-otros.

\section{III.4.4. Diferencias en función de los es- tudios cursados}

Respecto de los estudios cursados, únicamente en el factor "ocio compartido" $\{F(3,713)=3,06 ; p<0,03\}$ y en el factor de "asesoramiento/orientación" $\quad\{\mathrm{F}(3,709)=$ $4,55 ; \mathrm{p}<0,004\}$, existen diferencias significativas (tabla 6). Concretamente en el primer caso, los participantes que no han cursado ningún tipo de estudio puntúan de forma significativamente inferior a todos los demás. Con relación al factor "asesoramiento/orientación", el grupo de sin estudios también puntúa de forma inferior con relación al grupo de estudios medios y al grupo de estudios superiores (tabla 6).

Tabla 6. Estudios cursados

\begin{tabular}{|c|c|c|c|c|c|c|c|c|c|c|c|}
\hline & \multicolumn{2}{|c|}{ Sin estudios } & \multicolumn{2}{|c|}{ E.G.B. } & \multicolumn{2}{|c|}{ Est. medios } & \multicolumn{2}{|c|}{ Est. Super. } & \multirow{2}{*}{$\mathrm{F}$} & \multirow{2}{*}{$\mathrm{p}$} & \multirow{2}{*}{$\eta^{2}$} \\
\hline Variables & $\mathrm{X}$ & Sx & $\mathrm{X}$ & $\mathrm{Sx}$ & $\bar{X}$ & $\mathrm{Sx}$ & $\bar{X}$ & Sx & & & \\
\hline$\overline{F_{1}}$ & 12,60 & 3,78 & 13,52 & 4,05 & 13,65 & 3,49 & 14,31 & 3,31 & 2,25 & 0,08 & 0,009 \\
\hline $\mathrm{F}_{2}$ & 15,20 & 3,73 & 16,28 & 3,22 & 16,27 & 3,02 & 15,91 & 2,97 & 1,34 & 0,26 & 0,006 \\
\hline $\mathrm{F}_{3}{ }^{\mathrm{ABC}}$ & 10,20 & 2,61 & 11,66 & 2,43 & 11,54 & 2,24 & 11,65 & 2,28 & 3,06 & 0,03 & 0,01 \\
\hline $\mathrm{F}_{4}{ }^{\mathrm{BC}}$ & 11,16 & 3,22 & 12,19 & 2,65 & 12,70 & 2,17 & 12,57 & 2,20 & 4,55 & 0,004 & 0,02 \\
\hline$\overline{F_{5}}$ & 13,84 & 2,59 & 14,20 & 1,66 & 14,15 & 1,66 & 14,06 & 1,74 & 0,48 & 0,70 & 0,002 \\
\hline
\end{tabular}

$X=$ Media; $S x=$ Desviación típica; $\mathrm{F}=$ Prueba de contraste de hipótesis $\mathrm{F}$ Ratio; $\mathrm{p}=$ probabilidad; $\eta^{2}=$ tamaño del efecto; $F_{1}=$ Factor de "Implicación escolar"; $F_{2}=$ Factor de "Dedicación personal"; $F_{3}=$ Factor de "Ocio compartido"; $F_{4}=$ Factor de "Asesoramiento/orientación" y $F_{5}=$ Factor de "Asunción del rol de ser padre/madre". Prueba de Tukey: ${ }^{A}$ Sin estudios-E.G.B.; ${ }^{B}$ Sin estudios-Estudios medios; ${ }^{C}$ Sin estudios -Estudios superiores.

\section{DISCUSIÓN}

La ECCP-p presenta una estructura factorial adecuada, ya que pretende evaluar cómo se perciben los/as padres/madres a través de cin- co dimensiones consideradas por diferentes autores como competencia parental (Baumrind, 1968, 1971; Cerón y Gallardo, 2002; García, 1.994; Inglés, 1.995; Molpeceres y cols., 1994; Moreno, Jiménez, Oliva, Palacios 
y Saldaña, 1.995 Musitu y Cava, 2001; Villar, Luengo, Gómez y Romero, 2003).

Los datos obtenidos confirman que la consistencia interna de la escala es alta $(a=0,86)$, así como en cada una de las cinco subescalas. Aunque sea de forma tentativa, también presenta una adecuada validez discriminante. Además, tanto la distribución de los items como la distribución multivariada cumplen los criterios de normalidad.

Con relación al primer factor (implicación escolar), muchos padres y madres creen que no tienen por qué intervenir en las tareas y conocimientos que desde el colegio se establecen. Piensan que es responsabilidad de los profesores. Esta idea se ve reforzada por los datos presentados en octubre de 2003 por el Instituto Nacional de Evaluación y Calidad (INCE, 2003. Citado en http://www.consumer.es/ -julio de 2004-), en el que revelan que la mayoría de las familias españolas concede a la educación de sus hijos/as un papel relevante; no obstante, prefieren vivir ausentes ante lo que ocurre en las aulas. Es más, únicamente el 2,30\% de los/as padres y madres españolas participan en el proceso de aprendizaje de sus hijos/as y se implica en las tareas que realizan en el colegio (INCE, 2003).

Este hecho es totalmente contraproducente, ya que se ha señalado con frecuencia que la participación activa de los/as padres/madres en la escuela incide de forma positiva en el rendimiento académico, las habilidades sociales, la autoestima, el menor absentismo, los hábitos de estudio y las actitudes positivas hacia la escuela de los/as hijos/as. También los/as padres/madres mejoran de esta forma sus actitudes hacia la escuela, hacia el profesorado y hacia sí mismos. Cuando la comunicación familia-escuela es fluida, los profesores perciben también una mayor competencia en sus actividades y un mayor compromiso con el currículo académico y con el niño/a (Musitu y Cava, 2001).

Esta serie de aspectos están contenidos en la ECCP-p. A modo de ejemplo, el item 11 "mantengo un contacto frecuente con la escuela para estar al tanto de cualquier información referen- te a los programas y recursos para las familias", refleja este hecho.

Respecto del factor "dedicación personal" evalúa en qué medida los/as padres/madres dedican sus tiempos y espacios para conversar, aclarar dudas, etc. (item 10: "Ayudo a mis hijos/as a establecer una rutina diaria en cuanto a hábitos de higiene"). Los/as hijos/as asumen actitudes de aceptación y rechazo frente a las expectativas y demandas de los/as padres/madres en función de variables tales como su percepción acerca de la legitimidad de la autoridad paterna. Molpeceres y cols. (1994) han señalado una serie de variables que influyen en el menor o mayor éxito de los/as padres/madres en la transmisión de valores a los/as hijos/as, tales como el grado de control materno y/o paterno en relación con los estándares culturales, el ámbito en el que se produce el intento de control, la adecuación de la disciplina al tipo de problema, el estilo de disciplina del padre y/o de la madre, el interés generacional y la motivación de los/as hijos/as para identificarse con sus padres y sus madres, etc.

El factor "ocio compartido" (item 7 "veo con mis hijos/as ciertos programas de TV y los comento con ellos/as después") analiza a la familia como un agente socializador. Es decir, se trata de una institución importante para que los/as hijos/as aprendan a conocer el medio en el que viven y a integrarse en él. En este aspecto las familias tienen una responsabilidad ineludible en la educación y formación de los/as hijos/as y el ocio forma parte de ese proceso, en virtud del cual se forma la personalidad.

El factor "asesoramiento/orientación" (item 16 "existe una hora fija en la que mis hijos/as tengan que estar acostados o levantados". Item 17 "dedico un tiempo al día para hablar con mi hijo/a") destaca la capacidad de diálogo y de comunicación de los/as padres/madres ante las demandas y necesidades de los/as hijos/as. Cuando existe la comunicación en una familia, seguramente se puede afirmar que existe un compañerismo, una complicidad, y un ambiente de unión y afecto. Habrá sobretodo un respeto mutuo y unos valores más asentados. Sin em- 
bargo, crear este clima de comunicación en la familia, no es así una tarea tan fácil. Hay que ayudar a los/as hijos/as con prácticas, es decir, que los/as padres/madres introduzcan mecanismos que faciliten la comunicación. Es más, los datos evidencian que los adolescentes que consumen drogas, o se involucran en otras conductas problemáticas, perciben a su familia como distante y poco unida, y a sus padres y a sus madres como menos involucrados en sus actividades y con patrones de comunicación deficientes (Baer y Bray, 1999; Baumrind, 1991; Villar et al., 2003).

El factor "asunción del rol de ser madre/padre" (item 22 "soy muy consciente del cambio que ha experimentado mi familia con la llegada de los/as hijos/as") pretende medir hasta qué punto los progenitores se han adaptado a las circunstancias que conlleva el nacimiento de un hijo/a. Hay padres y madres que tienen dificultades para adaptarse a la nueva situación, y la afrontan delegando las responsabilidades en otros como pareja, abuelos, etc. Es decir, presentan dificultades para cambiar sus hábitos $\mathrm{y}$, como consecuencia, pueden convertirse en padres y madres negligentes. La negligencia familiar hacia los/as hijos/as es una de las tipologías detectadas con mayor frecuencia en nuestro país y su impacto en la población infantil no es menos severa que el maltrato físico, pues conlleva en muchas ocasiones secuelas emocionales y físicas, como se ha detectado en diferentes trabajos (Inglés, 1.995; Moreno, Jiménez, Oliva, Palacios y Saldaña, 1.995; Villar et al., 2003).

Es de sobra conocido que, con anterioridad a la aplicación de una intervención, un aspecto indispensable es el de realizar un diagnóstico de la situación en la que se encuentran las personas implicadas. Tras los resultados obtenidos, la ECPP-p cumple dicha función.

Es nuestro deseo que este instrumento de medida se convierta en una herramienta útil para que los profesionales de los servicios educativos, sociales, así como de otros ámbitos, sean capaces de detectar situaciones en las que se precise una intervención encaminada a mejorar la competencia parental como moduladora de conductas problemáticas en los/as adolescentes.

No obstante, aunque la ECPP-p reúne unas propiedades psicométricas adecuadas, ya que permite evaluar a la gran mayoría de padres/madres con hijos/as en edad escolar su competencia parental, es necesario analizar la estabilidad temporal de la escala, y si la estructura factorial se replica con muestras más elevadas y con diferentes grupos en situación de exclusión social. Además, habría que contrastar las puntuaciones obtenidas en la ECPP-p con medidas objetivas. De esta forma, se obtendría de una forma más adecuada su validez y su fiabilidad.

\section{REFERENCIAS}

Aguilar Ramos, M ${ }^{\mathrm{a}}$ C. (2002). Educación familiar: una propuesta disciplinar y curricular. Málaga: Ediciones Aljibe.

Baer, P. E. \& Bray, J. H. (1999). Adolescent individuation and alcohol use. Journal of Studies on Alcohol, Supl. 13, 52-62.

Baumrind, D. (1968). Authorizian vs. authoritative parental control. Adolescence, 3, 255-272.

Baumrind, D. (1971). Current patterns of parental authority. Developmental Psychology Monographs, 4(1), 1.102.

Baumrind, D. (1991). The influence of parenting style on adolescent competence and substance use. Journal of Early Adolescence, 11(1), 56-95.

Bersabé, R. M., Fuentes, M. J. y Motrico, E. (2001). Análisis psicométrico de dos escalas para evaluar estilos educativos parentales. Psicothema, vol. 13, $\mathrm{n}^{\circ}$ 4, 678-684.

Castro, J.; Toro, J.; Van Der Ende, J. \& y Arrindel, W. A. (1993). Exploring the feasibility of assessing perceived parental rearing styles in spanish children with the EMBU. International Journal of Social Psychiatry, 39, 47-57.

Ceballos, E. y Rodrigo, M.J. (1992). Cuestionario Situacional de Metas y Prácticas Educativas. La Laguna: Servicio de Publicaciones de la Universidad de la Laguna.

Cerezo, M. A., Keesler, T. Y. Dunn. E.S. y Wahler, R.G. (1991). Standardiced Observation Codes III. En M. A Cerezo (Ed.), Interacciones 
Bayot, A., Hernández Viadel, J. V. y de Julian, Luis Felipe (2005): Análisis factorial exploratorio y propiedades psicométricas de la escala de competencia parental percibida. Versión para padres/madres (ECPP-p). RELIEVE: v. 11, n. 2, p. 113-126. http://www.uv.es/RELIEVE/v11n2/RELIEVEv11n2 2.htm

familiares: un sistema de evaluación observacional (pp.17-59). Madrid: Mepsa.

Eysenck, H. J. (1976). Delincuencia y personalidad. Madrid: Marova, S.L.

Fariña, F.; Seijo, D.; Novo, M. y Jólluskin, G. (2002). Instrumentos específicos de evaluación forense (IEEF) en Derecho de familia. Comunicación presentada en el congreso virtual organizado por Interpsiquis. Febrero de 2002. www.psiquiatria.com/congreso.

Fuentes, M. J., Motrico, E. y Bersabé, R. M. (1999). Escala de Afecto (EA) y Escala de Normas y Exigencias (ENE): Versión hijos y versión padres. Málaga: Universidad de Málaga.

Gerard, A. B. (1998). Parent-child relationship inventory (PCRI). Los Ángeles: Western Psychological Services

Gracia, E. y Musitu, G. (1993). El maltrato infantil. Un análisis ecológico de los factores de riesgo. Madrid: Ministerio de Asuntos Sociales.

Grisso, T. (1986). Evaluating Competencies: Forensic Assessments and Instruments. New York: Plenum Press.

Hereford, C. F. (1963). Changing parental attitudes through group discussion. Austin: University of Texas Press.

Holden, G. \& Edwards, I. (1989). Parental attitudes toward child rearing: instruments, issues, and implications. Psychological Bulletin, 106,29-58.

Inglés, A. (1995). Origen, proceso y algunos resultados del estudio sobre los malos tratos infantiles en Cataluña. Infancia y Aprendizaje, $71,23-32$.

Instituto Nacional de Evaluación y Calidad (2003). Citado en http://www.consumer.es/web/es/noticias/educa cion y ciencia//2003/10/14//66523.php

Magaz, A. y García, E. (1998). PPE, Perfil de Estilos Educativos. Baracaldo: Consultores en Ciencias Humanas.

Martínez, J. L., Fuertes, A., Ramos, M. y Hernández, A. (2003). Consumo de drogas en la adolescencia: importancia del afecto y la supervisión parental. Psicothema, 15 (2), 161166.

Molpeceres, M. A.; Musitu, G. y Lila, M. (1994). La socialización del sistema de valores en el ámbito familiar. En G. Musitu y P. Allat (comp.), Psicosociología de la familia (pp. 121-146). Valencia: Albatros.

Moreno, M. C., Jiménez, J., Oliva, A., Palacios, J. y Saldaña, D. (1995). Detección y caracterización del maltrato infantil en la Comunidad Autónoma Andaluza. Infancia y Aprendizaje, $71,33-47$

Musitu, G. y Cava, M. J. (2001). La familia y la educación. Barcelona: Octaedro.

Palacios, J. (1994). Escala de evaluación de estilos Educativos (4E). Sevilla: Servicio de publicaciones de la Universidad de Sevilla.

Roa. L. y del Barrio, V. (2001). Adaptación del cuestionario de Crianza Parental (PCRI-M) a población española. Revista Latinoamericana de Psicología, 333, 324-341.

Villar, P.; Luengo, M ${ }^{\mathrm{a}}$ A.; Gómez, J. A. y Romero, E. (2003). Una propuesta de evaluación de variables familiares en la prevención de la conducta problema en la adolescencia. Psicothema, 15 (4), 581-588.

Wahler, R., Williams, A.J. and Cerezo, M.A. (1990). The compliance and predictability Hypothesis: sequential and correlational analyses of coercive mother-child Interactions. Behavioval Assement, 12, 391-407.

\section{AGRADECIMIENTOS}

El proyecto de investigación ha sido subvencionado por el "Plan Concertado para las Prestaciones de Servicios Sociales Básicas" (Ministerio de Trabajo y Asuntos Sociales, la Consejería de Bienestar Social de la Junta de Comunidades de Castilla-La Mancha y el Excmo Ayto. de Cuenca).

Además, queremos mostrar nuestro agradecimiento a D. José Manuel Martínez Cenzano, D. Javier Priego Moreno, D. Juan Manual Ávila Francés, D. José Ignacio Albentosa Hernández, Da Begoña del Pozo Herráiz, Da Elisa Hervías Arquero, D ${ }^{\mathrm{a}} \mathrm{M}^{\mathrm{a}}$ Amalia Sánchez Rubio y a $\mathrm{D}^{\mathrm{a}}$ Ana Isabel Valverde Martínez. 


\section{NOTAS}

[1] En el momento actual, el equipo de investigación está desarrollando la Escala de Competencia Parental Percibida, en su versión para hijos/as (ECPP-h).

\section{ABOUT THE AUTHORS / SOBRE LOS AUTORES}

Agustín Bayot (agustin.bayot@uclm.es). Profesor Titular del Área de Métodos de Investigación y Diagnóstico en Educación de la Universidad de Castilla-La Mancha. Su dirección postal es: Facultad de CC. de la Educación y Humanidades. Avda. de los Alfares, 44. 16071-Cuenca (España).

José Vicente Hernández Viadel (JoseVicente.Hernandez@uclm.es). Profesor asociado de la Escuela Universitaria de Magisterio, Universidad de Castilla-La Mancha. Experto en intervención familiar.

Luis Felipe de Julián (luisdejulian@hotmail.com). Becario de investigación. Doctorando en el programa "Cambio social y educación". 
Bayot, A., Hernández Viadel, J. V. y de Julian, Luis Felipe (2005): Análisis factorial exploratorio y propiedades psicométricas de la escala de competencia parental percibida. Versión para padres/madres (ECPP-p). RELIEVE: v. 11, n. 2, p. 113-126. http://www.uv.es/RELIEVE/v11n2/RELIEVEv11n2_2.htm

\title{
ARTICLE RECORD / FICHA DEL ARTÍCULO
}

\begin{tabular}{|c|c|}
\hline $\begin{array}{l}\text { Reference / } \\
\text { Referencia }\end{array}$ & $\begin{array}{l}\text { Bayot, Agustín, Hernández Viadel, José Vicente y de Julián, Luis Felipe (2005). Análisis facto- } \\
\text { rial exploratorio y propiedades psicométricas de la escala de competencia parental percibida. Ver- } \\
\text { sión para padres/madres (ECPP-p). Revista ELectrónica de Investigación y EValuación Educati- } \\
\text { va, v. 11, n. 2. http://www.uv.es/RELIEVE/v11n2/RELIEVEv11n2_2.htmConsultado en (poner } \\
\text { fecha). }\end{array}$ \\
\hline Title / Título & $\begin{array}{l}\text { Análisis factorial exploratorio y propiedades psicométricas de la escala de competencia parental } \\
\text { percibida. Versión para padres/madres (ECPP-p) [Exploratory factorial analysis and psychomet- } \\
\text { rics properties on the scale of sensed parental competencies. Version for parents (ECPP-p)] }\end{array}$ \\
\hline $\begin{array}{l}\text { Authors / } \\
\text { Autores }\end{array}$ & Agustín Bayot , José Vicente Hernández Viadel y Luis Felipe de Julián \\
\hline $\begin{array}{l}\text { Review / } \\
\text { Revista }\end{array}$ & Revista ELectrónica de Investigación y EValuación Educativa (RELIEVE), v. 11, n. 2 \\
\hline ISSN & $1134-4032$ \\
\hline $\begin{array}{l}\text { Publication } \\
\text { date / } \\
\text { Fecha de } \\
\text { publicación }\end{array}$ & $\begin{array}{l}2005 \text { (Reception Date: } 2005 \text { January 05; Approval Date: } 2005 \text { July 18; Publication Date: } \\
2005 \text { September 6) }\end{array}$ \\
\hline $\begin{array}{l}\text { Abstract / } \\
\text { Resumen }\end{array}$ & $\begin{array}{l}\text { The lack of parental (or relative) competence is related to the development of a model of unsuit- } \\
\text { able behaviour in children (sons or daughters), suffering the consequences of this fact. However, } \\
\text { practically there is no existence of evidences that can measure this. The scale of Parental Compe- } \\
\text { tence sensed (parents vision) (ECPP-P) it is composed by twenty-two items which are structured } \\
\text { in five factors. The ECPP shows some psychometric properties highly acceptables. } \\
\text { La escasa competencia parental está relacionada con el desarrollo de un patrón de conducta de- } \\
\text { sadaptativo en los/as hijos/as, con las consecuencias que este hecho conlleva. Sin embargo, prácti- } \\
\text { camente no existen pruebas que midan este constructo. La Escala de Competencia Parental Perci- } \\
\text { bida (versión para padres/madres)(ECPP-p) está constituida por } 22 \text { items, que se estructuran en } \\
\text { cinco factores. La ECPP presenta unas propiedades psicométricas altamente aceptables. }\end{array}$ \\
\hline $\begin{array}{l}\text { Keywords } \\
\text { Descriptores }\end{array}$ & $\begin{array}{l}\text { Parental competence, familiar interaction, parental satisfaction, problematic behaviours. } \\
\text { Competencia parental, interacción familiar, satisfacción parental, conductas problemáticas }\end{array}$ \\
\hline $\begin{array}{l}\text { Institution / } \\
\text { Institución }\end{array}$ & Universidad de Castilla - La Mancha (España) \\
\hline $\begin{array}{l}\text { Publication } \\
\text { site / } \\
\text { Dirección }\end{array}$ & http://www.uv.es/RELIEVE \\
\hline $\begin{array}{l}\text { Language / } \\
\text { Idioma }\end{array}$ & Español (Title, abstract and keywords in english ) \\
\hline
\end{tabular}

\section{Revista ELectrónica de Investigación y $\mathbf{E V}$ aluación $\mathbf{E}$ ducativa (RELIEVE)}

\author{
[ ISSN: 1134-4032 ]
}

\begin{abstract}
(C) Copyright, RELIEVE. Reproduction and distribution of this articles it is authorized if the content is no modified and their origin is indicated (RELIEVE Journal, volume, number and electronic address of the document).

(C) Copyright, RELIEVE. Se autoriza la reproducción y distribución de este artículo siempre que no se modifique el contenido y se indique su origen (RELIEVE, volumen, número y dirección electrónica del documento).
\end{abstract}

